\title{
Magnetic Field Effect on Nitrogenation of $\mathrm{Sm}_{2} \mathrm{Fe}_{17}$
}

\author{
Keiichi Koyama $^{1, *}$, Masahira Onoue ${ }^{1}$, Ryota Kobayashi ${ }^{1}$, Yoshifuru Mitsui ${ }^{1}$, Rie Y. Umetsu ${ }^{2}$ and \\ Yoshiya Uwatoko ${ }^{3}$
}

${ }^{1}$ Graduate School of Science and Engineering, Kagoshima University, Kagoshima 890-0065, Japan

${ }^{2}$ Institute for Materials Research, Tohoku University, Sendai 980-8577, Japan

${ }^{3}$ Institute for Solid State Physics, The University of Tokyo, Kashiwa 277-8581, Japan

\begin{abstract}
Nitrogenation of $\mathrm{Sm}_{2} \mathrm{Fe}_{17}$ powder was performed under a zero field and a magnetic field of $5 \mathrm{~T}$ at 623,673 and $743 \mathrm{~K}$ to clarify the magnetic field effect on nitrogenation. Applying a magnetic field of $5 \mathrm{~T}$ induced nitrogenation compared with zero-field nitrogenation, and almost fully nitride $\mathrm{Sm}_{2} \mathrm{Fe}_{17} \mathrm{~N}_{2.9}$ was obtained at $743 \mathrm{~K}$. Mössbauer spectroscopy results suggested that a 5-T magnetic field promoted the phase transformation to the fully-nitride $\mathrm{Sm}_{2} \mathrm{Fe}_{17} \mathrm{~N}_{3}$ phase. The magnetic field effect was discussed based on the magnetic energy gain and magnetic properties of host $\mathrm{Sm}_{2} \mathrm{Fe}_{17}$ and fully nitride $\mathrm{Sm}_{2} \mathrm{Fe}_{17} \mathrm{~N}_{3}$. [doi:10.2320/matertrans.MT-MN2019012]
\end{abstract}

(Received December 20, 2019; Accepted March 26, 2020; Published May 22, 2020)

Keywords: magnetic field effect, nitrogenation, gas-solid reaction, $\mathrm{Sm}_{2} \mathrm{Fe}_{17} \mathrm{~N}_{3}$

\section{Introduction}

Ferromagnetic (FM) nitride $\operatorname{Sm}_{2} \mathrm{Fe}_{17} \mathrm{~N}_{x}(0<x \leq 3)$ with a rhombohedral $\mathrm{Th}_{2} \mathrm{Zn}_{17}$-type structure (space group $R \overline{3} m$ ) was synthesized using the $\mathrm{N}_{2}$ gas-solid reaction technique on host $\mathrm{Sm}_{2} \mathrm{Fe}_{17 .}{ }^{1)}$ The host's cell volume expands by $6 \%-7 \%$ to accommodate three nitrogen atoms at the 9e-interstitial sites. $^{2)}$ The fully-nitride (FN) $\mathrm{Sm}_{2} \mathrm{Fe}_{17} \mathrm{~N}_{3}$ has high Curie temperature $T_{\mathrm{C}}$ of $746 \mathrm{~K}$, high saturation magnetization $\mu_{0} M_{\mathrm{S}}$ of $1.51 \mathrm{~T}$ and large anisotropy field of $21 \mathrm{~T}$ at room temperature (RT). ${ }^{3)}$ Therefore, $\mathrm{FN} \mathrm{Sm}_{2} \mathrm{Fe}_{17} \mathrm{~N}_{3}$ is a candidate for permanent magnets. ${ }^{2,4)}$ However, $\mathrm{Sm}_{2} \mathrm{Fe}_{17} \mathrm{~N}_{3}$ decomposes to $\alpha$-Fe and $\mathrm{SmN}$ above $720 \mathrm{~K}^{4,5)}$

Magnetic field stabilizes the FM phase by gaining large magnetic energy (Zeeman energy) $G_{\mathrm{M}}$. For example, magnetic fields induced $\mathrm{FM}$ phases of $\mathrm{LaCo}_{5} \mathrm{H}_{x}$, 6 ,7) $\mathrm{MnBi}^{8-12)}$ and $\mathrm{MnAl} .{ }^{13,14)}$ Therefore, it is expected that magnetic fields will promote nitrogenation for $\mathrm{Sm}_{2} \mathrm{Fe}_{17}$. However, to the best of our knowledge, the magnetic field effect (MFE) on nitrogenation of the $\mathrm{Sm}_{2} \mathrm{Fe}_{17}$ system had not been reported in detail. Recently, to clarify the MFE on nitrogenation, we developed an in-field heat treatment furnace utilized for a 5-T cryocooled superconducting magnet with a $50 \mathrm{~mm}$ room-temperature bore. ${ }^{15)}$ In this paper, we describe a review of MFE on the nitrogenation of $\mathrm{Sm}_{2} \mathrm{Fe}_{17}$, which suggests competition between enhancement of phase transformation and suppression of diffusion processes by applying a magnetic field.

\section{Experimental Procedure}

The host $\mathrm{Sm}_{2} \mathrm{Fe}_{17}$ compound was prepared by induction melting of the constituent elements under an argon atmosphere and then annealed at $1423 \mathrm{~K}$ for $24 \mathrm{~h}$ in an argon atmosphere for homogeneity treatment. ${ }^{16)}$ The $\mathrm{Sm}_{2} \mathrm{Fe}_{17}$ ingot was crushed and pulverized into powder with particle size less than $53 \mu \mathrm{m}$ in diameter. The lattice parameters $a$ and $c$ of $\mathrm{Sm}_{2} \mathrm{Fe}_{17}$ were $0.856 \mathrm{~nm}$ and $1.24 \mathrm{~nm}$, respectively. The zerofield nitrogenation (ZFN) and in-field nitrogenation (IFN) of

*Corresponding author, E-mail: koyama@sci.kagoshima-u.ac.jp
$5 \mathrm{~T}$ (IFN-5T) for the $\mathrm{Sm}_{2} \mathrm{Fe}_{17}$ powder were performed under a nitrogen gas pressure $P_{\mathrm{n}}$ of $0.1 \mathrm{MPa}$ at nitrogenation temperature $T_{\mathrm{n}}$ of 623,673 and $743 \mathrm{~K}$ for 24 hours in an applied magnetic field $\mu_{0} H$ of $5 \mathrm{~T}$. The IFN equipment is described in Ref. 15) in detail. The nitrogen content $x$ in the nitrided powders was estimated from the increased sample mass after nitrogenation. Detailed sample preparation is reported in Ref. 17).

To examine the phases of ZFN and IFN-5T samples, X-ray powder diffraction (XRD) measurements were performed using $\mathrm{Cu}-\mathrm{K} \alpha$ radiation at RT. Magnetization $M$ data were corrected using a superconducting quantum interference device magnetometer and a vibrating sample magnetometer. $T_{\mathrm{C}}$ of the sample was determined by differential scanning calorimetry under a zero field. ${ }^{57} \mathrm{Fe}$ Mössbauer spectroscopy experiments with a $1.85 \mathrm{GBq}{ }^{57} \mathrm{Co}(\mathrm{Rh})$ source were performed using a conventional constant acceleration method at RT. The velocity scale was calibrated with $\alpha$-Fe which had a hyperfine field of $33.1 \mathrm{~T}$. The values of the hyperfine parameters were refined using NORMOS. ${ }^{18)}$

\section{Results}

The estimated $x$ in the $\mathrm{Sm}_{2} \mathrm{Fe}_{17} \mathrm{~N}_{x}$ powder prepared by ZFN and IFN-5T are listed in Table 1. In this table, the data for host $\mathrm{Sm}_{2} \mathrm{Fe}_{17}$ are also shown. It is clear that $x$ of IFN-5T is larger than that of ZFN for all $T_{\mathrm{n}}$. By applying a magnetic field of $5 \mathrm{~T}, x$ increased by $0.4-0.6$ nitrogen atoms per formula unit. For condition of $T_{\mathrm{n}}=743 \mathrm{~K}$, almost $\mathrm{FN}$ $\mathrm{Sm}_{2} \mathrm{Fe}_{17} \mathrm{~N}_{2.9}$ was obtained using IFN-5T, while $x$ was only 2.3 using ZFN. Magnetic field promotes nitrogenation for the temperature $T$ range of $623-743 \mathrm{~K}$. From XRD analysis for ZFN and IFN-5T samples, ${ }^{17)}$ we confirmed that the poornitride (PN) and FN phase coexisted in the nitride sample with $x \leq 1.9$. However, the XRD patterns for $x=2.3$ and 2.9 did not show clearly the two phase coexistence of the PN and FN phases but a single nitride phase with a very small amount of $\alpha$-Fe phase. $\left.{ }^{15}\right)$ The determined lattice parameters for the nitride with $x=2.3$ and 2.9 are listed in Table 1 . The parameters of the nitride with $x=2.9$ are comparable to the data for $\mathrm{FN} \mathrm{Sm}_{2} \mathrm{Fe}_{17} \mathrm{~N}_{3}$ reported in previous literature. ${ }^{2)}$ 
Table 1 Conditions of zero-field nitrogenation (ZFN) and $5 \mathrm{~T}$ in-field nitrogenation (IFN-5T) for 24h, nitrogen content $x$, lattice parameters, Curie temperature, relative fraction of poor-nitride phase $(\mathrm{PN})$ and fully-nitride phase $(\mathrm{FN})$, and average hyperfine field $\left\langle B_{\mathrm{hf}}\right\rangle$.

\begin{tabular}{|c|c|c|c|c|c|c|c|c|c|}
\hline & \multirow{2}{*}{$\begin{array}{l}T_{\mathrm{n}} \\
(\mathrm{K})\end{array}$} & \multirow[t]{2}{*}{$x$} & \multirow{2}{*}{$\begin{array}{c}a \\
(\mathrm{~nm})\end{array}$} & \multirow{2}{*}{$\begin{array}{c}c \\
(\mathrm{~nm})\end{array}$} & \multirow{2}{*}{$\begin{array}{r}T_{\mathrm{C}} \\
(\mathrm{K})\end{array}$} & \multicolumn{2}{|c|}{ rel. fraction } & \multicolumn{2}{|c|}{$<B_{\mathrm{hP}}>$} \\
\hline & & & & & & $\mathrm{PN}$ & $\mathrm{FN}$ & $\mathrm{PN}(\mathrm{T})$ & $\mathrm{FN}(\mathrm{T})$ \\
\hline ZFN & 623 & 0.6 & \multicolumn{2}{|c|}{ two phase } & two phase & - & - & - & - \\
\hline IFN-5T & 623 & 1.0 & \multicolumn{2}{|c|}{ two phase } & two phase & - & - & - & - \\
\hline ZFN & 673 & 1.3 & \multicolumn{2}{|c|}{ two phase } & two phase & - & - & - & - \\
\hline IFN-5T & 673 & 1.9 & \multicolumn{2}{|c|}{ two phase } & two phase & - & - & - & - \\
\hline ZFN & 743 & 2.3 & 0.874 & 1.27 & two phase & 0.157 & 0.843 & 25.5 & 33.3 \\
\hline IFN-5T & 743 & 2.9 & 0.874 & 1.27 & 746 & 0.082 & 0.918 & 25.7 & 33.2 \\
\hline $\mathrm{Sm}_{2} \mathrm{Fe}_{17}$ & - & - & 0.856 & 1.24 & 391 & 1 & 0 & 21.5 & - \\
\hline
\end{tabular}

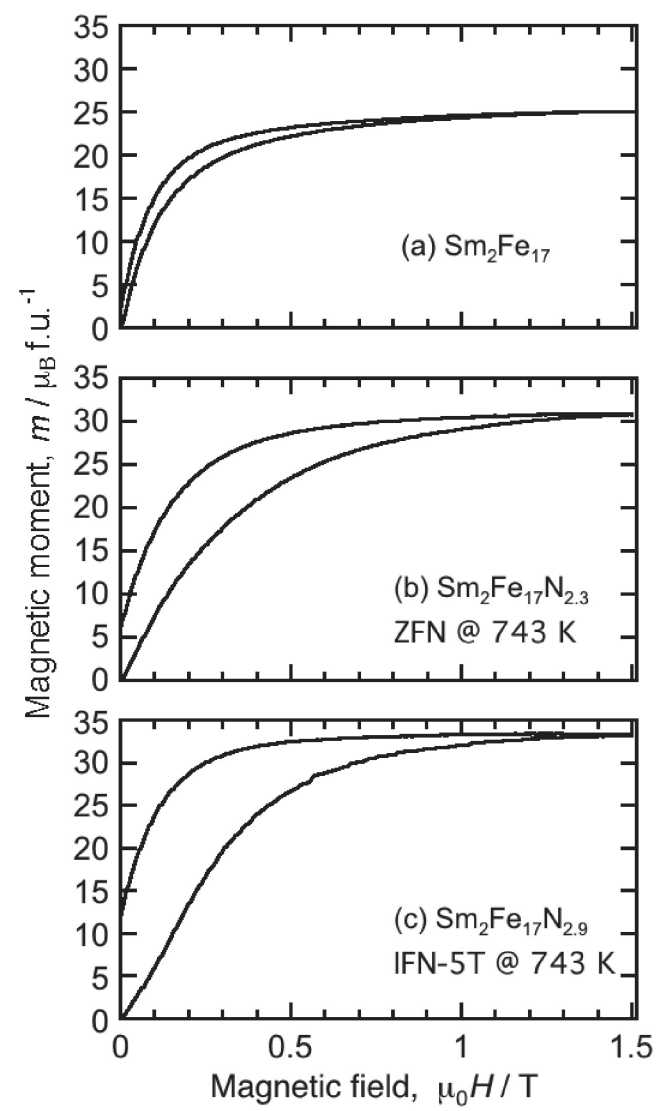

Fig. 1 Magnetization curves of $\mathrm{Sm}_{2} \mathrm{Fe}_{17}$ (a), $\mathrm{Sm}_{2} \mathrm{Fe}_{17} \mathrm{~N}_{2.3}$ (ZFN) (b) and $\mathrm{Sm}_{2} \mathrm{Fe}_{17} \mathrm{~N}_{2.9}$ (IFN-5T) (c) at room temperature.

Figure 1 shows the magnetization $(M-H)$ curves of $\mathrm{Sm}_{2} \mathrm{Fe}_{17}$ (a), $\mathrm{Sm}_{2} \mathrm{Fe}_{17} \mathrm{~N}_{2.3}$ (b) and $\mathrm{Sm}_{2} \mathrm{Fe}_{17} \mathrm{~N}_{2.9}$ (c) at RT. The saturation magnetic moments $m_{\mathrm{S}}$ were estimated to be $25.1 \mu_{\mathrm{B}} /$ f.u. for $\mathrm{Sm}_{2} \mathrm{Fe}_{17}, 31.3 \mu_{\mathrm{B}}$ /f.u. for $\mathrm{Sm}_{2} \mathrm{Fe}_{17} \mathrm{~N}_{2.3}$ and $33.8 \mu_{\mathrm{B}} /$ f.u. for $\mathrm{Sm}_{2} \mathrm{Fe}_{17} \mathrm{~N}_{2.9}$. The value of $m_{\mathrm{S}}$ for $\mathrm{Sm}_{2} \mathrm{Fe}_{17} \mathrm{~N}_{2.9}$ is comparable to that for $\mathrm{FN} \mathrm{Sm}_{2} \mathrm{Fe}_{17} \mathrm{~N}_{3}$. ${ }^{2)} \mathrm{We}$ confirmed that the magnetic moment $m$ and remanence of the IFN-5T sample (Fig. 1(c)) are larger than those of the ZFN sample (Fig. 1(b)). IFN-5T induced the hard magnetic properties of $\mathrm{Sm}_{2} \mathrm{Fe}_{17} \mathrm{~N}_{x}$.

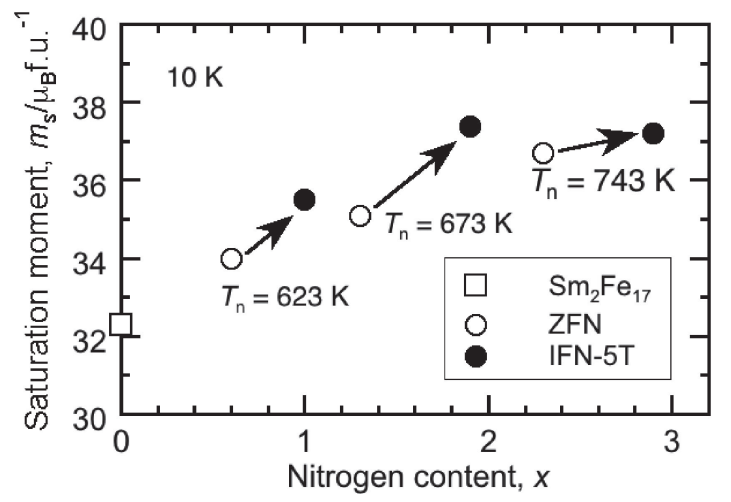

Fig. 2 Saturation moment for ZFN, IFN-5T and $\mathrm{Sm}_{2} \mathrm{Fe}_{17}$ at $10 \mathrm{~K}$.

The thermomagnetization $(M-T)$ curves of host $\mathrm{Sm}_{2} \mathrm{Fe}_{17}$, $\mathrm{Sm}_{2} \mathrm{Fe}_{17} \mathrm{~N}_{2.3}$ (ZFN) and $\mathrm{Sm}_{2} \mathrm{Fe}_{17} \mathrm{~N}_{2.9}$ (IFN-5T) in a magnetic field of $1 \mathrm{~T}$ were measured in the $T$ range of $10-830 \mathrm{~K}$, which was shown in Ref. 15). The $M-T$ curves for $0.6 \leq x \leq 2.3$ did not show the Brillouin-function like curve but a superposition of two $M-T$ curves, ${ }^{15)}$ suggesting that the PN and FN phase coexisted in the sample. The $M-T$ curve of $\mathrm{Sm}_{2} \mathrm{Fe}_{17} \mathrm{~N}_{2.9}$ showed a single Brillouin-function curve. ${ }^{15)}$ The determined $T_{\mathrm{C}}$ of $\mathrm{Sm}_{2} \mathrm{Fe}_{17} \mathrm{~N}_{2.9}$ was $746 \mathrm{~K}$, which is almost the same as that of $\mathrm{FN} \mathrm{Sm}_{2} \mathrm{Fe}_{17} \mathrm{~N}_{3}$. ${ }^{2)}$ Figure 2 shows $m_{\mathrm{S}}$ at $10 \mathrm{~K}$ for the ZFN (open circles) and IFN-5T (solid circles). The magnetic field of $5 \mathrm{~T}$ enhanced the nitrogen content $x$ and magnetic properties of $\mathrm{Sm}_{2} \mathrm{Fe}_{17} \mathrm{~N}_{x}$ at each $T_{\mathrm{n}}$.

Figure 3 shows the Mössbauer spectra of $\mathrm{Sm}_{2} \mathrm{Fe}_{17}$, $\mathrm{Sm}_{2} \mathrm{Fe}_{17} \mathrm{~N}_{2.3}$ and $\mathrm{Sm}_{2} \mathrm{Fe}_{17} \mathrm{~N}_{2.9}$ at RT. In this figure, dots and solid curves indicate experimental data and fitting curves, respectively. Since Fe atoms occupy $6 \mathrm{c}, 9 \mathrm{~d}, 18 \mathrm{f}$ and $18 \mathrm{~h}$ sites in the $\mathrm{Th}_{2} \mathrm{Zn}_{17}$-type structure, the spectrum of $\mathrm{Sm}_{2} \mathrm{Fe}_{17}$ was fitted with four sub-spectra using hyperfine interaction parameters: site-occupation, isomer shift, quadruple splitting and hyperfine field $B_{\mathrm{hf}}$. The determined average $B_{\mathrm{hf}},\left\langle B_{\mathrm{hf}}\right\rangle$, was $21.5 \mathrm{~T}$ for $\mathrm{Sm}_{2} \mathrm{Fe}_{17}$, which is in good agreement with reported data $(21.8 \mathrm{~T})$ for $\mathrm{Sm}_{2} \mathrm{Fe}_{17}{ }^{2)}$ The spectra of $\mathrm{Sm}_{2} \mathrm{Fe}_{17} \mathrm{~N}_{2.3}$ and $\mathrm{Sm}_{2} \mathrm{Fe}_{17} \mathrm{~N}_{2.9}$ were fitted with eight subspectra due to the $\mathrm{PN}$ and $\mathrm{FN}$ phases. The determined parameters of relative fraction and $\left\langle B_{\mathrm{hf}}\right\rangle$ of $\mathrm{Sm}_{2} \mathrm{Fe}_{17} \mathrm{~N}_{2.3}$ and 


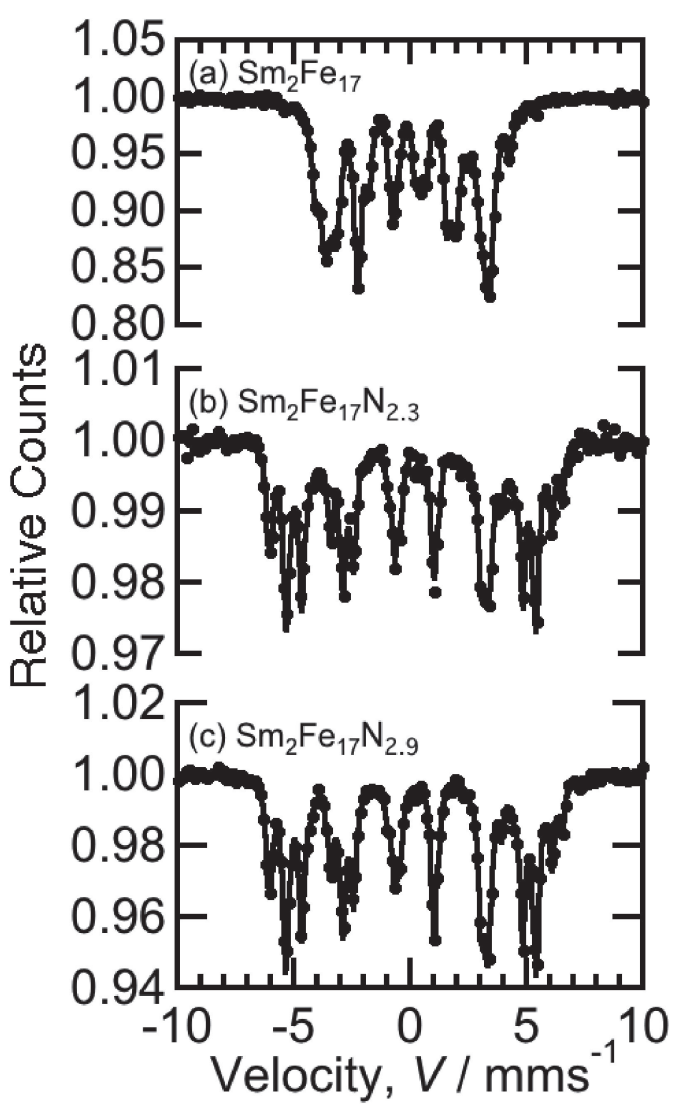

Fig. 3 Mössbauer spectra of $\mathrm{Sm}_{2} \mathrm{Fe}_{17}$ (a), $\mathrm{Sm}_{2} \mathrm{Fe}_{17} \mathrm{~N}_{2.3}$ (ZFN) (b) and $\mathrm{Sm}_{2} \mathrm{Fe}_{17} \mathrm{~N}_{2.9}$ (IFN-5T) (c) at room temperature.

$\mathrm{Sm}_{2} \mathrm{Fe}_{17} \mathrm{~N}_{2.9}$ were shown in Table 1. The relative fraction of the FN phase of $x=2.9$ (IFN-5T) was $92 \%$, which is larger than that of $x=2.3$ (ZFN). In contrast, the relative fraction of the PN phase of IFN-5T is smaller than that of ZFN. $\left\langle B_{\mathrm{hf}}\right\rangle$ of the PN phase was determined to be $25.7 \mathrm{~T}$ for $x=2.3$ (ZFN) and $24.9 \mathrm{~T}$ for $x=2.9$ (IFN-5T), which were larger than that of $\mathrm{Sm}_{2} \mathrm{Fe}_{17}$. The determined $\left\langle B_{\mathrm{hf}}\right\rangle$ of the FN phase was 33.3$33.4 \mathrm{~T}$, which is in good agreement with reported data $(33.3 \mathrm{~T})$ of $\mathrm{Sm}_{2} \mathrm{Fe}_{17} \mathrm{~N}_{3} .{ }^{19)}$ From Mössbauer spectroscopy experiments, it was found that both $\mathrm{PN}$ and $\mathrm{FN}$ phases existed in the nitrides with $x=2.3$ and 2.9. The obtained results indicate that $m_{\mathrm{s}}$ of the PN phase is smaller than that of the FN phase but slightly larger than that of $\mathrm{Sm}_{2} \mathrm{Fe}_{17}$.

The results of this study showed that magnetic fields promoted phase transformation from the $\mathrm{PN}$ to the FN phase under at same $T_{\mathrm{n}}$. That is, the equilibrium state of the $\mathrm{N}_{2}$ gassolid reaction probably was changed by applying a magnetic field to form the FN phase with large $m$.

\section{Discussion}

In this section, MFE on the nitridation of $\mathrm{Sm}_{2} \mathrm{Fe}_{17}$ is discussed. The gain of $G_{\mathrm{M}}, \Delta G_{\mathrm{M}}$, can be driving force of the gas-solid reaction. $\Delta G_{\mathrm{M}}$ of the Gibbs free energy tends to be more stable of a FM state with large $m$ and promotes ferromagnet synthesis. So far, it has been reported that the reaction or phase transformations from the non-FM to FM phase was accelerated by magnetic fields. ${ }^{10,11,14,20)}$

Our results showed the two-phase coexistence of the FN and $\mathrm{PN}$ phases in $\mathrm{Sm}_{2} \mathrm{Fe}_{17} \mathrm{~N}_{x}(x<3)$, so that the field-

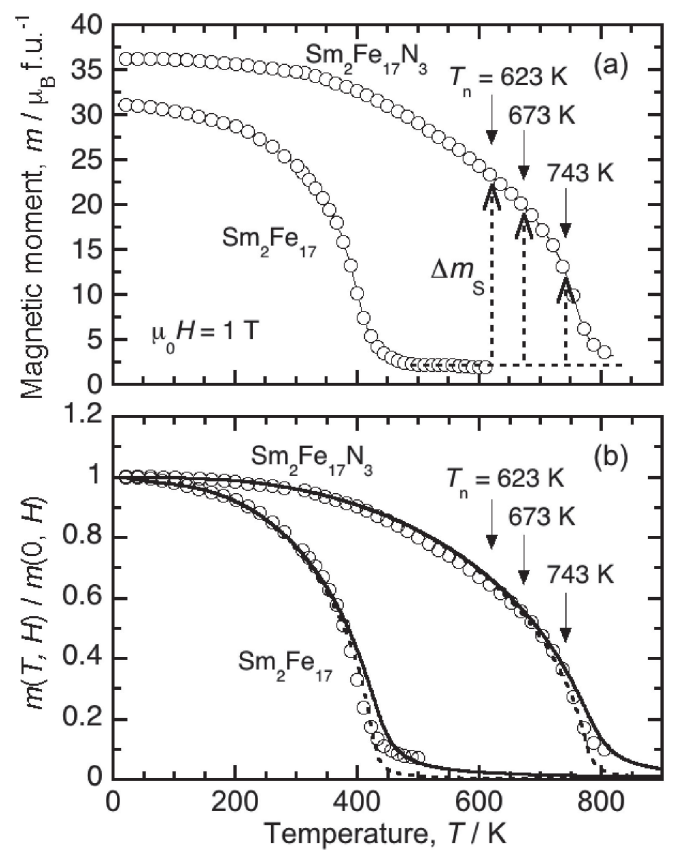

Fig. 4 Temperature dependence of the magnetic moments (a) and calculated moment $m(T, H) / m(0, H)$ (b) of $\mathrm{Sm}_{2} \mathrm{Fe}_{17}$ and $\mathrm{Sm}_{2} \mathrm{Fe}_{17} \mathrm{~N}_{3}$ for $\mu_{0} H=1 \mathrm{~T}$ (broken curve) and $5 \mathrm{~T}$ (solid curve). The open circles present the experimental data of $\mu_{0} H=1 \mathrm{~T}$.

induced phase transformation by $\Delta G_{\mathrm{M}}$ is a possible mechanism. Assuming a simple model with only the Zeeman effect, $\Delta G_{\mathrm{M}}$ under $H$ can be written as

$$
\Delta G_{M}=\mu_{0} H \Delta m_{\mathrm{S}},
$$

where $\mu_{0}$ is the permeability of a vacuum and $\Delta m_{\mathrm{S}}$ is the change of the saturation moment. ${ }^{21,22)}$ In the present case, $\Delta m_{\mathrm{S}}$ is expressed by

$$
\Delta m_{\mathrm{S}}=m_{\mathrm{PN}}-m_{\mathrm{FN}},
$$

where $m_{\mathrm{PN}}$ and $m_{\mathrm{FN}}$ are the magnetic moments for the PN and FN phases, respectively. The MFE direction is found by the sign of $\Delta m_{\mathrm{S}}{ }^{22}$ ) As shown in Fig. 3 of Ref. 15), FN $\mathrm{Sm}_{2} \mathrm{Fe}_{17} \mathrm{~N}_{3}$ is ferromagnetic, but the $\mathrm{PN}$ phase is paramagnetic at $T_{\mathrm{n}}=623 \mathrm{~K}, 673 \mathrm{~K}$ and $743 \mathrm{~K}$, so that the sign of $\Delta m_{\mathrm{S}}$ and $\Delta G_{\mathrm{M}}$ is negative in this $T_{\mathrm{n}}$ range. This indicates that the MFE promotes the phase transformation from the PN to $\mathrm{FN}$ phase. ${ }^{22)}$

It is difficult to evaluate magnetic moment of the $\mathrm{PN}$ phase in the $M$ - $T$ curve of $\mathrm{Sm}_{2} \mathrm{Fe}_{17} \mathrm{~N}_{x}(x<3)$ sample because of a superposition of two $M-T$ curves. $^{15)}$ Since present IFN was conducted under a magnetic field of $5 \mathrm{~T}$, we estimated $m(T, H)$ of $\mathrm{Sm}_{2} \mathrm{Fe}_{17}$ as the PN phase and $\mathrm{Sm}_{2} \mathrm{Fe}_{17} \mathrm{~N}_{3}$ as the FN phase for $\mu_{0} H=5 \mathrm{~T}$ based on a mean field theory for a

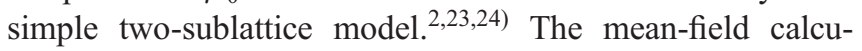
lation is described in the appendix.

Figure 4 shows the temperature dependence of the magnetic moments (a) and calculated moment $m(T, H)$ / $m(0, H)$ (b) of $\mathrm{Sm}_{2} \mathrm{Fe}_{17}$ and $\mathrm{Sm}_{2} \mathrm{Fe}_{17} \mathrm{~N}_{3}$ for $\mu_{0} H=1 \mathrm{~T}$ (broken curves) and $5 \mathrm{~T}$ (solid curves). In this calculation, the effective exchange parameters were $J^{\mathrm{FeFe}}=290 \mathrm{~K}$ and $J^{\mathrm{SmFe}}=10 \mathrm{~K}$ for $\mathrm{Sm}_{2} \mathrm{Fe}_{17}$, and $J^{\mathrm{FeFe}}=540 \mathrm{~K}$ and $J^{\mathrm{SmFe}}=$ $50 \mathrm{~K}$ for $\mathrm{Sm}_{2} \mathrm{Fe}_{17} \mathrm{~N}_{3}$. The open circles in Fig. 4 show the experimental data collected under $\mu_{0} H=1 \mathrm{~T}{ }^{15)}$ The 


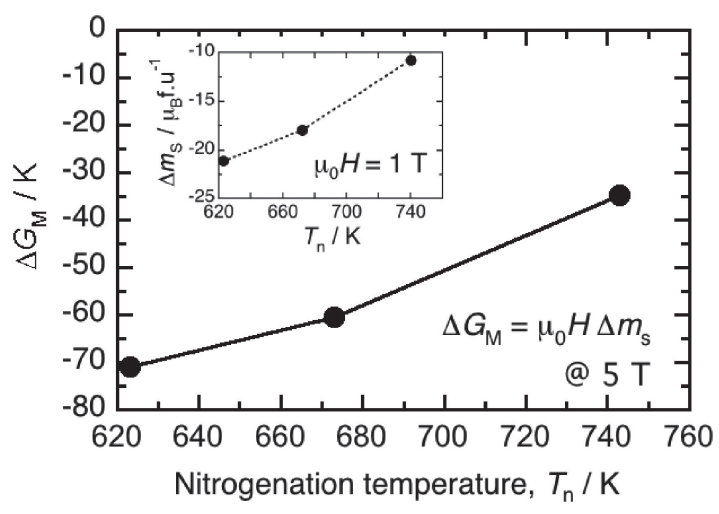

Fig. 5 Nitrogenation temperature dependence of the change in magnetic energy $\Delta G_{\mathrm{M}}$ under $\mu_{0} H=5 \mathrm{~T}$, which was evaluated using eq. (1) and the change in magnetic moment $\Delta m_{\mathrm{S}}$. The inset shows $\Delta m_{\mathrm{S}}$ deduced from Fig. 4(a).

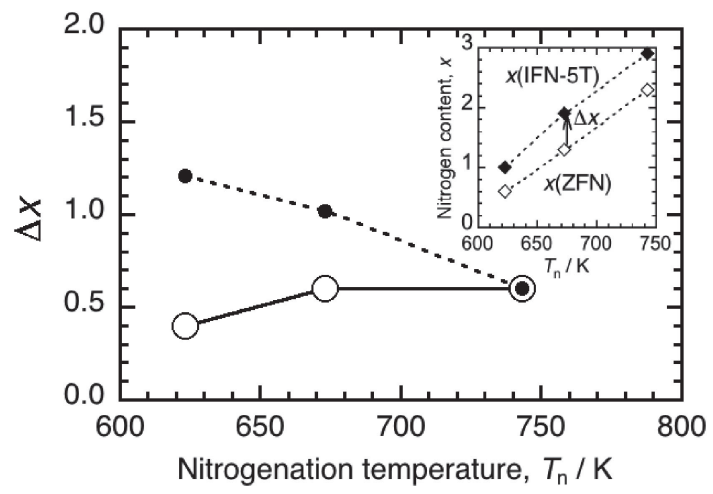

Fig. 6 Field-induced nitrogen content $\Delta x$ as a function of nitrogenation temperature $T_{\mathrm{n}}$. The open circles indicate experimental $\Delta x$ deduced from $\Delta x=x($ IFN-5T $)-x(\mathrm{ZFN})$. The solid circles indicate $\Delta x$ estimated by only $\Delta G_{\mathrm{M}}$. The inset shows that nitrogen content $x$ as function of $T_{\mathrm{n}}$ for ZFN (open diamonds) and IFN-5T (solid diamonds).

calculated $m-T$ curves represent the experimental data. The deference between the experimental data of $\mu_{0} H=1 \mathrm{~T}$ and calculated data of $\mu_{0} H=5 \mathrm{~T}$ is small. Therefore, we considered that the field-induced $m$ was negligibly small within the present IFN-5T condition, and $m_{\mathrm{PN}}$ and $m_{\mathrm{FN}}$ under $\mu_{0} H=5 \mathrm{~T}$ were regarded as the experimental $m$ of $\mathrm{Sm}_{2} \mathrm{Fe}_{17}$ and $\mathrm{Sm}_{2} \mathrm{Fe}_{17} \mathrm{~N}_{3}$ for $\mu_{0} H=1 \mathrm{~T}$, respectively. Since $\Delta G_{\mathrm{M}}$ of an electron spin of $1 \mu_{\mathrm{B}}$ in $\mu_{0} H=1 \mathrm{~T}$ corresponds to thermal energy of $0.67 \mathrm{~K},{ }^{21)} T_{\mathrm{n}}$ dependence of $\Delta G_{\mathrm{M}}$ for $\mu_{0} H=5 \mathrm{~T}$ was obtained, as shown in Fig. 5, which was evaluated using eqs. (1) and (2). Here, $\Delta m_{\mathrm{S}}$ was deduced from Fig. 4(a) (broken arrows), which is presented in the inset of Fig. 5. With decreasing $T_{\mathrm{n}}$ from $T_{\mathrm{n}}=743 \mathrm{~K}, \Delta G_{\mathrm{M}}$ decreases: the gain of $G_{\mathrm{M}}$ becomes larger.

In Fig. 6, the field-induced nitrogen content $\Delta x$ is shown as function of $T_{\mathrm{n}}$. Here, the experimental $\Delta x$ (open circles) was evaluated from the inset of Fig. 6: $\Delta x=x($ IFN-5T) $x(\mathrm{ZFN})$. If the field-induced nitrogen content $(\Delta x=0.6)$ from $x(\mathrm{ZFN})=2.3$ to $x($ IFN-5T $)=2.9$ is only due to $\Delta G_{\mathrm{M}}$ (approximately $35 \mathrm{~K}$ ) at $T_{\mathrm{n}}=743 \mathrm{~K}$, the change of the nitrogen content $x$ was calculated to be $\Delta x=1.0$ at $T_{\mathrm{n}}=$ $673 \mathrm{~K}$ and $\Delta x=1.2 T_{\mathrm{n}}=623 \mathrm{~K}$, as shown by the solid circles in Fig. 6. The experimental $\Delta x$ at $T_{\mathrm{n}}=623 \mathrm{~K}$ and $673 \mathrm{~K}$ were smaller than $\Delta x$ estimated from $\Delta G_{\mathrm{M}}$. This result suggests that only phase transformation mechanism from the paramagnetic PN phase to ferromagnetic FN phase is hard to explain the field-induced nitrogenation. As seen in Fig. 6, the nitrogenation due to the field-induced phase transformation is probably suppressed at lower $T_{\mathrm{n}}$. We should consider other MFE mechanisms as well as the field-induced phase transformation in $\mathrm{Sm}_{2} \mathrm{Fe}_{17} \mathrm{~N}_{x}$ powder.

Fujii et al. reported that the FN-phase grain growth (phase transformation) becomes dominant at the later stage in nitrogenation at $733 \mathrm{~K}$ under $P_{\mathrm{n}} \geq 0.1 \mathrm{MPa}$, exceeding the nitrogen diffusion process, which is important at the earlier stage. ${ }^{16)}$ That is, at first, nitrogen atoms diffuse into the $\mathrm{Sm}_{2} \mathrm{Fe}_{17}$ powder from the surface, which forms the PN phase. After that, the PN phase transforms the FN phase. This means that the amount of the PN phase is important for progressing the grain growth of the FN phase (phase transformation).

It was reported that the carbon diffusion in $\gamma$-iron ${ }^{25)}$ and the diffusion process in $\mathrm{Fe} / \mathrm{Ga}$ diffusion couples $^{26}$ ) were suppressed by applying magnetic fields. If the nitrogen diffusion process for forming the PN phase is suppressed by applying a magnetic field, the competition of MFE between the enhancement of the phase transformation and suppression of the diffusion processes will occur. The nitrogen diffusion process is regarded as an activation type, ${ }^{4,16,27,28)}$ so that thermal effect of the diffusion is dominant at higher $T_{\mathrm{n}}$ such as $743 \mathrm{~K}$, compared with the nitrogen diffusion process suppressed by MFE. In contrast, the field-induced suppression of the nitrogen diffusion process becomes more effective at lower $T_{\mathrm{n}}$ such as $623 \mathrm{~K}$. This leads to smaller $\Delta x$ than that estimated from the phase transformation from the PN to FN phase by $\Delta G_{\mathrm{M}}$. Now, further IFN experiments in fields up to $15 \mathrm{~T}$ are in progress to verify this scenario, which will be reported soon elsewhere. However, it should be noted that the present results clearly show the promotion of nitrogenation of the $\mathrm{Sm}_{2} \mathrm{Fe}_{17}$ magnet by applying a magnetic field under our IFN condition.

\section{Conclusions}

ZFN and IFN-5T for $\mathrm{Sm}_{2} \mathrm{Fe}_{17}$ powder were conducted under nitrogen gas pressure of $0.1 \mathrm{MPa}$ at 623,673 and $743 \mathrm{~K}$ for $24 \mathrm{~h}$ to investigate the magnetic field effect on the nitrogenation to $\mathrm{Sm}_{2} \mathrm{Fe}_{17} \mathrm{~N}_{x}$. Nitrogenation of the $\mathrm{Sm}_{2} \mathrm{Fe}_{17}$ magnet was promoted by applying a magnetic field. Mössbauer spectroscopy results suggested that magnetic fields promoted the phase transformation from the poornitride to fully-nitride $\mathrm{Sm}_{2} \mathrm{Fe}_{17} \mathrm{~N}_{3}$ phase. It was hard to explain the magnetic field effect on nitrogenation in $\mathrm{Sm}_{2} \mathrm{Fe}_{17}$ by only mechanism of the field-induced phase transformation due to gain of magnetic free energy.

\section{Acknowledgment}

The authors are grateful to Prof. H. Fujii of Hiroshima University for providing the $\mathrm{Sm}_{2} \mathrm{Fe}_{17}$ host compound. This work was partly supported by KAKENHI $17 \mathrm{H} 00289$ and Iketani Science and Technology Foundation 0301019-A. Magnetization measurements were performed at the Institute for Materials Research, Tohoku University and the Institute for Solid State Physics, the University of Tokyo, Japan. 
Mössbauer spectroscopy experiments were carried out at the Division of Isotope Science, Research Support Center, Institute for Research Promotion, Kagoshima University.

\section{Appendix}

In the simple model, $m(T, H)$ is expressed by

$$
m(T, H)=2 m_{\mathrm{Sm}}(T, H)+17 m_{\mathrm{Fe}}(T, H),
$$

where $m_{\mathrm{Sm}}(T, H)$ and $m_{\mathrm{Fe}}(T, H)$ are the magnetic moments of $\mathrm{Sm}$ and $\mathrm{Fe}$ atoms, respectively. Then, $m_{\mathrm{Sm}}(T, H)$ and $m_{\mathrm{Fe}}(T, H)$ are written by

$$
m_{\mathrm{Sm}}(T, H)=g\langle J\rangle \mu_{\mathrm{B}}
$$

and

$$
m_{\mathrm{Fe}}(T, H)=2\langle S\rangle \mu_{\mathrm{B}},
$$

where $g$ is the Lande g-factor, $\langle J\rangle$ and $\langle S\rangle$ are thermal averages of the total angular momentum $J$ of Sm and the total spin momentum $S$ of Fe, respectively. The molecular field $H_{\mathrm{m}}$ acting on $m_{\mathrm{Sm}}(T, H)$ and $m_{\mathrm{Fe}}(T, H)$ can be written by

$$
\begin{aligned}
& H_{\mathrm{m}}(\mathrm{Sm})=\frac{(g-1)^{2}}{g \mu_{B}} J^{\mathrm{SmSm}} z^{\mathrm{SmSm}}\langle J\rangle
\end{aligned}
$$

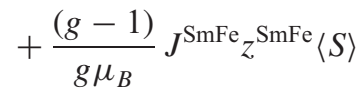

and

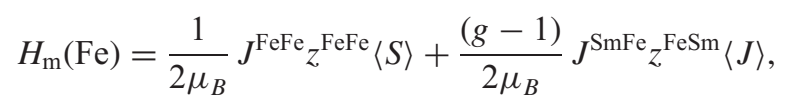

respectively. Here, $J^{\mathrm{SmSm}}, J^{\mathrm{SmFe}}$ and $J^{\mathrm{FeFe}}$ are the effective exchange parameters of the $\mathrm{Sm}-\mathrm{Sm}, \mathrm{Sm}-\mathrm{Fe}$ and $\mathrm{Fe}-\mathrm{Fe}$ interaction, respectively, and $z^{\mathrm{ij}}$ is the number of nearest neighbor j-atoms of an i-atom. In the $\mathrm{Th}_{2} \mathrm{Zn}_{17}$-type structure, Sm occupies the $6 \mathrm{c}$ site and Fe occupies the $6 \mathrm{c}, 9 \mathrm{~d}, 18 \mathrm{f}$ and $18 \mathrm{~h}$ sites. $z^{\mathrm{ij}}$ was determined to be $z^{\mathrm{SmSm}}=0, z^{\mathrm{SmFe}}=6$ $(\mathrm{Sm}-\mathrm{Fe}(18 \mathrm{f}))$ and $z^{\mathrm{FeSm}}=1(\mathrm{Fe}(18 \mathrm{f})-\mathrm{Sm})$. For four Fe sites, the average number of nearest neighbor $\mathrm{Fe}$ atoms of the $\mathrm{Fe}(6 \mathrm{c})-\mathrm{Fe}(6 \mathrm{c})$ (one bond), $\mathrm{Fe}(9 \mathrm{~d})-\mathrm{Fe}(18 \mathrm{f})$ (four bonds), $\mathrm{Fe}(18 \mathrm{f})-\mathrm{Fe}(9 \mathrm{~d})$ (two bonds) and $\mathrm{Fe}(18 \mathrm{~h})-\mathrm{Fe}(9 \mathrm{f})$ (two bonds) was estimated to be 2.2, and $z^{\mathrm{FeFe}}=2.2$ was adopted in this calculation.

It was assumed that the crystalline electric field energy (magnetocrystalline anisotropy energy) is negligibly small in $G_{\mathrm{m}}$ for the $T_{\mathrm{C}}$ and $T_{\mathrm{n}}$ range, compared with $J^{\mathrm{FeFe}}$ in this calculation. The ground $J=5 / 2$ multiplet and $g=0.286$ for $\mathrm{Sm}^{3+}$ ion was only considered and $S=1$ was adopted for the Fe-sublattice.

\section{REFERENCES}

1) J.M.D. Coey and H. Sun: J. Magn. Magn. Mater. 87 (1990) L251L254.

2) H. Fujii and H. Sun: Handbook of Magnetic Materials, ed. by K.H.J. Buschow, Vol. 9, (Elsevier, Amsterdam, 1995) pp. 310-353.

3) M. Katter, J. Wecker, C. Kuhrt, L. Schultz and R. Grössinger: J. Magn. Magn. Mater. 117 (1992) 419-427.

4) J.M.D. Coey and P.A.I. Smith: J. Magn. Magn. Mater. 200 (1999) 405424.

5) J.M.D. Coey: J. Magn. Magn. Mater. 159 (1996) 80-89.

6) M. Yamaguchi, H. Nomura, I. Yamamoto, T. Ohta and T. Goto: Phys. Lett. A 126 (1987) 133-135.

7) M. Yamaguchi, I. Yamamoto, T. Goto and S. Miura: Phys. Lett. A 134 (1989) 504-506.

8) K. Koyama, T. Onogi, Y. Mitsui, Y. Nakamori, S. Orimo and K. Watanabe: Mater. Trans. 48 (2007) 2414-2418.

9) K. Koyama, Y. Mitsui, E.S. Choi, Y. Ikehara, E.C. Palm and K. Watanabe: J. Alloy. Compd. 509 (2011) L78-L80.

10) Y. Mitsui, K. Koyama and K. Watanabe: Mater. Trans. 54 (2013) 242245.

11) Y. Mitsui, R.Y. Umetsu, K. Koyama and K. Watanabe: J. Alloy. Compd. 615 (2014) 131-134.

12) D. Miyazaki, Y. Mitsui, R.Y. Umetsu, K. Takahashi, S. Uda and K. Koyama: Mater. Trans. 58 (2017) 720-723.

13) R. Kobayashi, Y. Mitsui, R.Y. Umetsu, K. Takahashi, M. Mizuguchi and K. Koyama: IEEE Magn. Lett. 8 (2017) 1400704.

14) R. Kobayashi, Y. Mitsui, R.Y. Umetsu, K. Takahashi, M. Mizuguchi and K. Koyama: Mater. Trans. 58 (2017) 1511-1518.

15) M. Onoue, R. Kobayashi, Y. Mitsui, R.Y. Umetsu, Y. Uwatoko and K. Koyama: Mater. Trans. 60 (2019) 2179-2182.

16) H. Fujii, K. Tatami and K. Koyama: J. Alloy. Compd. 236 (1996) 156164.

17) M. Onoue, R. Kobayashi, Y. Mitsui, R.Y. Umetsu, Y. Uwatoko and K. Koyama: J. Alloy. Compd. (2020) in press.

18) R.A. Brand: WinNormos-for-Igor Users Manual Version 3.0 (2009). Available: http://physics-astronomy-manuals.wwu.edu/WinNormos3Manual.pdf.

19) K. Kobayashi, M. Ohmura, Y. Yoshida and M. Sagawa: J. Magn. Magn. Mater. 247 (2002) 42-54.

20) R. Onodera, S. Kimura, K. Watanabe, Y. Yokoyama, A. Makino and K. Koyama: J. Alloy. Compd. 604 (2014) 8-11.

21) M. Yamaguchi: Magneto-Science, ed. by M. Yamaguchi and Y. Tanimoto, (Springer, Berlin, 2007) pp. 1-8.

22) M. Yamaguchi: Magneto-Science, ed. by M. Yamaguchi and Y. Tanimoto, (Springer, Berlin, 2007) pp. 141-150.

23) N. Iwata, K. Hattori and T. Shigeoka: J. Magn. Magn. Mater. 53 (1986) 318-322.

24) E. Belorizky, M.A. Fremy, J.P. Gavigan, D. Givord and H.S. Li: J. Appl. Phys. 61 (1987) 3971-3973.

25) S. Nakamichi, S. Tsurekawa, Y. Morizono, T. Watanabe, M. Nishida and A. Chiba: J. Mater. Sci. 40 (2005) 3191-3198.

26) Y. Mitsui, T. Hagio, K. Takahashi and K. Koyama: ISIJ Int. 60 (2020) 807-811.

27) K. Kobayashi, K. Kaneko and K. Hayakawa: J. Alloy. Compd. 408412 (2006) 1363-1367.

28) R. Skomski and J.M.D. Coey: J. Appl. Phys. 73 (1993) 7602-7611. 